\title{
A Simple and General Proof of Beal's Conjecture (I)
}

\section{Golden Gadzirayi Nyambuya}

Department of Applied Physics, National University of Science and Technology, Bulawayo, Republic of Zimbabwe

Email: golden.nyambuya@nust.ac.zw

Received 15 July 2014; revised 15 August 2014; accepted 1 September 2014

Copyright (C) 2014 by author and Scientific Research Publishing Inc.

This work is licensed under the Creative Commons Attribution International License (CC BY).

http://creativecommons.org/licenses/by/4.0/

c) (i) Open Access

\section{Abstract \\ Using the same method that we used in [1] to prove Fermat's Last Theorem in a simpler and truly marvellous way, we demonstrate that Beal's Conjecture yields-in the simplest imaginable manner, to our effort to prove it.}

\section{Keywords}

Fermat's Last Theorem, Beal's conjecture, Proof

\section{Introduction}

"Simplicity is the ultimate sophistication."-Leonardo da Vinci (1452-1519).

Beal's Conjecture is a conjecture in number theory formulated in 1993 while investigating generalizations of Fermat's Last Theorem set forth in 1997 as a Prize Problem by the United States of America's Dallas, Texas number theory enthusiast and billionaire banker, Mr. Daniel Andrew Beal [2]. As originally stated, the conjecture asserts that:

\section{Beal's Conjecture:}

If,

$$
A^{x}+B^{y}=C^{z}
$$

$A, B, C, x, y$, and $z$ are positive integers with $(x, y, z)>2$, then $A, B$, and $C$ have a common prime factor.

For a correct proof or counterexample published in an internationally renowned and refereed mathematics journal, Mr. Beal initially offered a Prize of US\$5000.00 in 1997, raising it to $\$ 50000.00$ over ten years by 
adding US\$5000.00 each year over the ten-year period [2]. Very recently, Andrew Beal upped the stacks and has raised ${ }^{1}$ it beyond the initial projection of US\$50000.00 to US\$1000000.00.

Herein, we lay down a complete proof of the conjecture not so much for the very "handsome”, prize money attached to it, but more for the sheer intellectual challenge that the philanthropist-Mr. Andrew Beal, has placed before humanity. We believe that challenges without flinching-must be tackled heard-on, without fear of failure.

From intuition, we strongly believe or feel that a direct proof of the original statement of Beal conjecture as stated in (1) would be difficult if not impossible to procure. We have to recast this statement into an equivalent form and proceed to a proof by way of contradiction. The equivalent statement to (1) is [2]:

\section{Beal's Conjecture (Recast):}

The equation,

$$
A^{x}+B^{y}=C^{z},
$$

admits no solutions for any positive integers $A, B, C, x, y$, and $z$ with $(x, y, z)>2$ for any piecewise coprime triple $A, B$, and $C$.

In its recast form (2), it becomes clear that Beal's conjecture is a generalization of Fermat's Last Theorem [3] where Fermat's Last Theorem is the special case of Beal's conjecture where $x=y=z=n$. In the parlance of mathematics, Beal's conjecture is a corollary to Fermat's Last Theorem.

The proof that we present demonstrates that the triple $(A, B, C)$ can not be co-prime. This is the same method that we used in our "simple, and much more general Proof of Fermat's Last Theorem"' [1]. Actually, the present proof is a generalization of the proof of Fermat's Last Theorem presented in [1].

The synopsis of this reading is as follows. In $\S(2)$, we provide a pivotal lemma that is necessary for our argument. In $\S(3)$, we provide the short proof of Beal's Conjecture and thereafter in $\S(4)$, we give a general discussion and the conclusion drawn thereof.

\section{Lemma}

If $\left(a>1 ; a_{j} \leq a ; b>1 ; c>1 ; n>2\right) \in \mathbb{N}^{+}$where $(b>c)$ and $a_{j}$ is one of the prime factors of $a$, then, the following will hold true always:

$$
a^{n}=a_{j}(b+c) \text { or } a^{n}=a_{j}(b-c) .
$$

The above statement is clearly evident and needs no proof. Below we demonstrate that this statement is true. This demonstration does not constitute a proof.

What this statement really means is that the number $a^{n}$ (for any $n>2$ and $a>1$ ), can always be written as a sum or difference of two numbers $p$ and $q$ where $p \in \mathbb{N}^{+}$and $q \in \mathbb{N}^{+}$are not co-prime, i.e.:

$$
a^{n}=p+q \text { or } a^{n}=p-q: \operatorname{gcd}(p, q) \neq 1,
$$

since one can always find some $(p, q)$ such that $a$ will always be a common factor of $(p, q)$, that is to say:

$$
a^{n}=a(g+h) \text { or } a^{n}=a(g-h),
$$

in which case we will have $p=a g$ and $q=a h$ where $(g+h)=a^{n-1}>2$ such that $(g, h) \in \mathbb{N}^{+}$and $(g>h)$. If $\left\{a_{1}, a_{2}, a_{3}, \cdots, a_{j}, \cdots, a_{m}\right\}$ is the set of all the prime factors of $a$, then $a=a_{j} e$ where $e \leq a$. Substituting all this into (5), we will have:

$$
a^{n}=a_{j}(e g+e h) \text { or } a^{n}=a_{j}(e g-e h) .
$$

Setting $b=e g$ and $c=e h$, (6) leads us to (3). Therefore, equipped with this simple fact, we will demonstrate that as we did with Fermat's Last Theorem, that Beal's Conjecture yields to a proof in the simplest imaginable manner.

\footnotetext{
${ }^{1}$ The Beal Prize, AMS, http://www.ams.org/profession/prizes-awards/ams-supported/beal-prize
} 


\section{Proof}

The proof that we are going to provide is a proof by contradiction and this proof makes use of Lemma $\S(2)$ whereby we demonstrate that the triple $(x, y, z)$ is such that it will always have a common factor if the equation, $A^{x}+B^{y}=C^{z}$, for any $[(x, y, z)>2]$; is to hold true. We begin by assuming that the statement:

$$
A^{x}+B^{y}=C^{z} \text {, for any }[(x, y, z)>2]
$$

to be true for some piecewise co-prime triple $(A, B, C) \in \mathbb{N}^{+}$, the meaning of which is that the greatest common divisor of this triple or any arbitrary pair of the triple is unity i.e., $\operatorname{gcd}(A, B, C)=1$.

First, we must realise that if just one of the members of the triple $(A, B, C)$ is equal to unity for any $(x, y, z)>2$, then, the other two members of this triple can not be integers, hence, from this it follows that if a solution exists, then, all the members of this triple will be greater than unity i.e. $(A>1 ; B>1 ; C) \in \mathbb{N}^{+}$.

Now, for our proof, by way of contradiction, we assert that there exists a set of positive integers $(x, y, z)>2$ that satisfies the simple relation $A^{x}+B^{y}=C^{z}$ for some piecewise co-prime triple $(A, B, C)>1$. Having made this assumption, if we can show that $\operatorname{gcd}(A, B, C)>1$, then, by way of contradiction Beal's Conjecture holds true.

If the statement (7) holds true, then-clearly; there must exist some $(p, q) \in \mathbb{N}^{+}$such that $\operatorname{gcd}(p, q)=1$, such that $A^{x}, B^{y}$ and $C^{z}$ can be decomposed as follows:

$$
\left(\begin{array}{l}
A^{x} \\
B^{y} \\
C^{z}
\end{array}\right)=\left(\begin{array}{c}
p-q \\
2 q \\
p+q
\end{array}\right) .
$$

Now, according to the Lemma $\S(2)$, the equation $C^{z}=p+q$ for any $(z>2)$ and for any $(C>1)$, this equation, can always be written such that $p=a C_{j}$ and $p=b C_{j}$ for some $(a>1 ; b>1) \in \mathbb{N}^{+}$and $C_{j}:\left(1<C_{j} \leq C\right)$ is any of the prime factors of $C$; putting everything together, we will have $C^{z}=(a+b) C_{j}$. Substituting $p=a C_{j}$ and $q=b C_{j}$ into (8), we will have:

$$
\left(\begin{array}{l}
A^{x} \\
B^{y} \\
C^{z}
\end{array}\right)=\left(\begin{array}{c}
(a-b) C_{j} \\
2 b C_{j} \\
(a+b) C_{j}
\end{array}\right) .
$$

From (9), it is clear that $\operatorname{gcd}\left(A^{x}, B^{y}, C^{z}\right) \neq 1$ since there exists a common divisor $[\operatorname{cd}()]$ of the triple $\left(A^{x}, B^{y}, C^{z}\right)$ which is $\left(C_{j}>1\right)$, that is to say, $\left(C_{j}>1\right)$ is a common divisor of the triple $\left(A^{x}, B^{y}, C^{z}\right)$. If $\operatorname{gcd}\left(A^{x}, B^{y}, C^{z}\right) \neq 1$, consequently, $\operatorname{gcd}(A, B, C) \neq 1$ and this is in complete violation of the critical, crucial and sacrosanct assumption that $\operatorname{gcd}(A, B, C)=1$.

Alternatively, according to the Lemma $\S(2)$, the equation $A^{x}=p-q$ for any $(x>2)$ and for any $\left(A_{j}>1\right)$, this equation, can always be written such that $p=a A_{j}$ and $q=b a_{j}$ for some $(a>1 ; b>1) \in \mathbb{N}^{+}$and $A_{j}:\left(1<A_{j} \leq A\right)$ is any of the prime factors of $A$; putting everything together, we will have $A^{x}=(a-b) A_{j}$.

Now, substituting $p=a A_{j}$ and $q=b A_{j}$ into (8), we will have:

$$
\left(\begin{array}{l}
A^{x} \\
B^{y} \\
C^{z}
\end{array}\right)=\left(\begin{array}{c}
(a-b) A_{j} \\
2 b A_{j} \\
(a+b) A_{j}
\end{array}\right) .
$$

Again, from (10), it is clear that $\operatorname{gcd}\left(A^{x}, B^{y}, C^{z}\right) \neq 1$ since the $\operatorname{cd}\left(A^{x}, B^{y}, C^{z}\right)=x$, that is to say, $A_{j}$ is a common divisor of triple $\left(A^{x}, B^{y}, C^{z}\right)$. From the foregoing, it follows that the prime factors of $(A, C)$ are common divisors of the triple $\left(A^{x}, B^{y}, C^{z}\right)$, the meaning of which is that $\operatorname{gcd}(A, B, C) \neq 1$.

Therefore, by way of contradiction, Beal's Conjecture is true since we arrive at a contradictory result that $\operatorname{gcd}(A, B, C) \neq 1$. What this effectively means is that the equation $A^{x}+B^{y}=C^{z}$ for $(x, y, z)>2$ has a 
solution and this solution is such that the triple $(A, B, C)$ always has a common factor as is the case with all those values of $A, B, C$ that satisfy Beal's Conjecture.

\section{Discussion}

At present, it appears that there has not been found a general proof of Beal's conjecture, only partial solutions exist. For example, the case $(x, y, z)=(2,3,7)$ and all its permutations were proven to have only four solutions, none of them involving an even power greater than 2 by Poonen et al. [4]. The case $(x, y, z)=(2,3,8)$ and all its permutations are known to have only three solutions, none of them involving an even power greater than 2 and this was proved by Crandall \& Pomerance [5]. There are a number of such cases [6]-[8] where partial proofs have been presented. As-well, there are cases where computer searchers are made [9]. If Beal's is true as we have shown herein, then, all computer searches will never find a counter-example and the best way to resolved this would be via general proof as we have done here.

Our thrust has been on a direct proof and just as the proof presented in the reading [1], the proof here provided is simple, general and all-encompassing. It covers all possible cases. Clearly, the present proof applies elementary methods of arithmetic that where available even in the days of Fermat. At this point, if anything, we only await the judgement of the world of mathematics as to whether this proof is correct or not. Without any oversight on our confidence in our proof, allow us to say that, until such a time that evidence to the contrary is brought forth, we are at any rate, convinced of the correctness of the proof here presented.

We have presented another proof of Fermat's Last Theorem in the reading [10] and this proof makes use of the method of Pythagorean triples. This proof is much longer than the proof using the methods presented herein. We will be presenting a second version of the proof of Beal's Conjecture using the method of Pythagorean triples used in [10].

\section{Conclusion}

We hereby make the following conclusion that if our proof is correct as we strongly believe, then, Beal's Conjecture seizes to be a conjecture but forthwith transforms into a fully-fledged theorem as a logically and mathematically correct proof has now been supplied.

\section{References}

[1] Nyambuya, G.G. (2014) On a Simpler, Much More General and Truly Marvellous Proof of Fermat’s Last Theorem (I). http://vixra.org/abs/1309.0154

[2] Daniel Mauldin, R. (1997) A Generalization of Fermat's Last Theorem: The Beal Conjecture and Prize Problem. Notices of the American Mathematical Society, 44, 1436-1439.

[3] Wiles, A. (1995) Modular Elliptic Curves and Fermat’s Last Theorem. Annals of Mathematics, 141, 443-551. http://dx.doi.org/10.2307/2118559

[4] Poonen, B., Schaefer, E.F. and Stoll, M. (2007) Twists of X(7) and Primitive Solutions to $x^{2}+y^{3}=z^{7}$. Duke Mathematical Journal, 137, 103-158. http://dx.doi.org/10.1215/S0012-7094-07-13714-1

[5] Crandall, R. and Pomerance, C. (2000) Prime Numbers: A Computational Perspective. Spinger Science \& Business Media, Berlin, 147.

[6] Siksek, S. and Stoll, M. (2014) The Generalised Fermat Equation $x^{2}+y^{3}=z^{15}$. Archiv der Mathematik, 102, $411-421$. http://dx.doi.org/10.1007/s00013-014-0639-z

[7] Dahmen, S.R. and Siksek, S. (2014) Perfect Powers Expressible as Sums of Two Fifth or Seventh Powers. arXiv: 1309.4030v2.

[8] Darmon, H. and Granville, A. (1995) On the Equations $z^{\mathrm{m}}=F(x, y)$ and $A x^{\mathrm{p}}+B y^{\mathrm{q}}=C z^{\mathrm{r}}$. Bulletin of the London Mathematical Society, 27, 513-543. http://dx.doi.org/10.1112/blms/27.6.513

[9] Thiagarajan, R.C. (2014) A Proof to Beal's Conjecture. Bulletin of Mathematical Sciences \& Applications, 89-93.

[10] Nyambuya, G.G. (2014) On a Simpler, Much More General and Truly Marvellous Proof of Fermat's Last Theorem (II). http://vixra.org/abs/1405.0023 
Scientific Research Publishing (SCIRP) is one of the largest Open Access journal publishers. It is currently publishing more than 200 open access, online, peer-reviewed journals covering a wide range of academic disciplines. SCIRP serves the worldwide academic communities and contributes to the progress and application of science with its publication.

Other selected journals from SCIRP are listed as below. Submit your manuscript to us via either submit@scirp.org or Online Submission Portal.
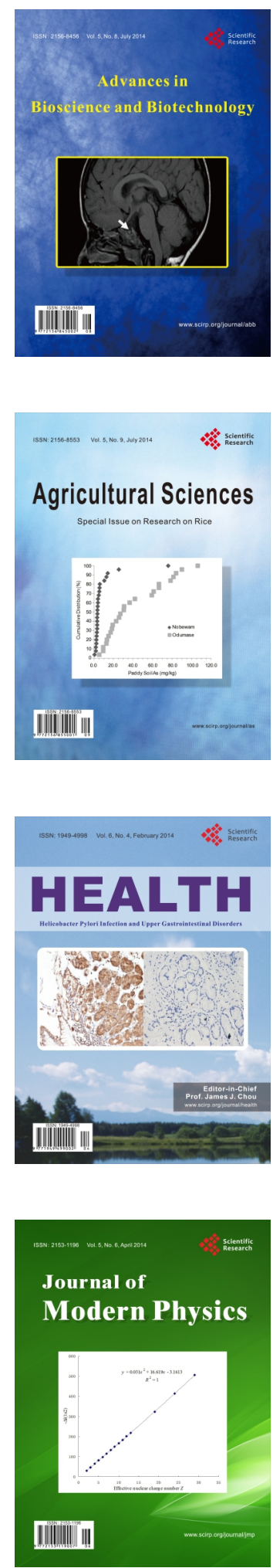
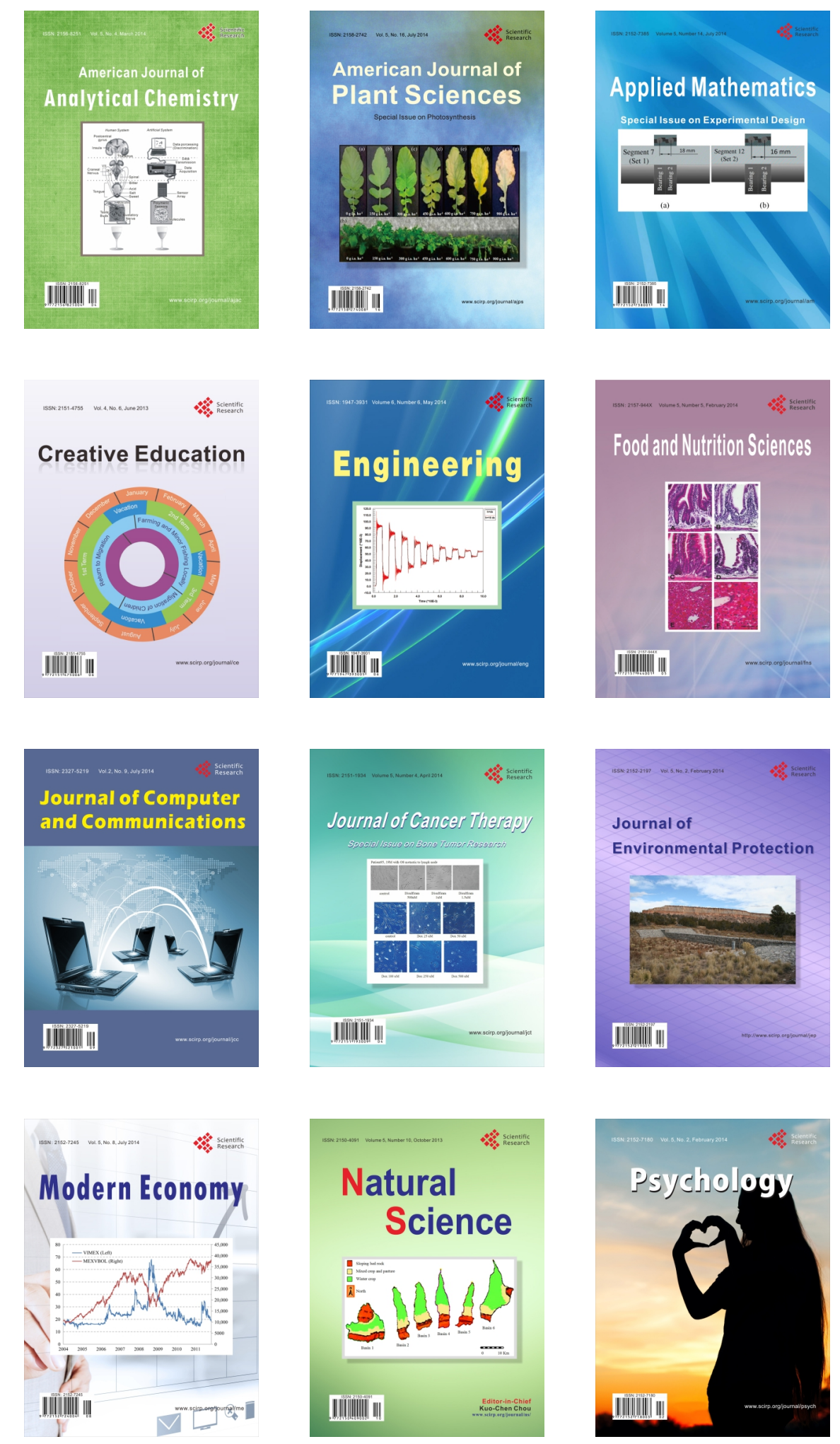